\title{
Does progesterone therapy increase nuchal translucency in women with threatened miscarriage?
}

\author{
Mehmet Keçecioğlu, Aytekin Tokmak, Tuğban S. Keçecioğlu, Burak Akselim, \\ Burcu K. Karakaya, Yasemin Taşçı
}

Dr. Zekai Tahir Burak Women's Health Education and Research Hospital, Ankara, Turkey

\begin{abstract}
Objectives: The effect of exogenous progesterone on fetal nuchal translucency (NT) has been proposed recently. In this study, we aimed to compare the thickness of NT of patients receiving and not receiving progesterone for threatened miscarriage. Material and methods: This study was designed as a retrospective comparative study. Ninety five women treated with progesterone constituted the study group whereas 97 women who were not treated with progesterone constituted the control group. An ultrasonographic examination was performed on all of the women to measure NT. All patients were treated with oral micronized progesterone in the study group. The main parameters recorded for each woman were; age, body mass index (BMI), obstetrical characteristics, and gestational age at first examination, treatment duration of progesterone therapy, and results of combined and triple tests.

Results: A total of 192 pregnant women with threatened miscarriage were included in this study. The mean NT thickness was statistically significantly higher in the study group $(p<0.001)$, and mean serum level of human chorionic gonadotropin (hCG) was also higher in this group $(p<0.05)$. There was no statistically significant difference between groups in terms of age, BMl, and gestational age at first examination. ROC curve analysis demonstrated that only increased NT (area under the curve: $0.634, \mathrm{p}=0.005,95 \% \mathrm{Cl}: 0.541-0.727$ ) was a discriminative factor for women receiving progesterone for threatened miscarriage. Also there was a positive correlation between NT and treatment duration $(r=0.269 ; p<0.001)$.

Conclusions: We think that oral progesterone therapy may increase NT depending on treatment duration without causing abnormal prenatal screening test results.
\end{abstract}

Key words: nuchal translucency, prenatal screening tests, progesterone therapy, threatened miscarriage

Ginekologia Polska 2016; 87, 5: 390-394

\section{INTRODUCTION}

Prenatal screening tests first appeared in the mid-1970s, with the use of maternal serum alpha-fetoprotein (AFP) levels for neural tube defects [1]. Then, the concept of the multiple of the median (MoM) value was proposed to standardize inter-laboratory variations and factors that may affect the serum AFP level, e.g. gestational age [2]. Obviously, pregnancies with certain fetal aneuploidies will cause psychological and financial loss in the affected families and communities. Therefore, various non-invasive screening tests have been developed for early detection and termination of these pregnancies, without harming the mother. Wald et al. [3], combined AFP, unconjugated estriol $\left(\mathrm{E}_{3}\right)$, and human chorionic gonadotropin (hCG) with maternal age to produce an estimate of the risk of having a Down syndrome pregnancy, and they called it the 'triple test'. This screening tool has been widely used all over the world until the end of the 90 s.

Serum levels of pregnancy-associated plasma protein-A (PAPP-A) and free $\beta$-hCG show significant changes in the mothers of aneuploid fetuses [4]. Increased thickness of fetal nuchal translucency (NT), defined as a transient fluid collection behind the fetal neck and visible on ultrasound at the end of the first trimester, is also a sensitive marker of fetal aneuploidy [5]. The combined first-trimester screening, which includes all of these parameters, was popular in the 2000s. Later, different risk assessment tests and integrated tests have been proposed. In the end, cell-free fetal nucleic acids in maternal serum have proceeded to take their place [6]. 
Abortion is a spontaneous loss of a fetus $<20$ weeks of gestation. It is a traumatic event, which can have psychological consequences for the parents [7]. Threatened miscarriage, demonstrated by vaginal bleeding with or without abdominal cramps, is a common complication of early pregnancy. It occurs in about a fifth of all pregnancies and approximately half of them will be lost. Progesterone is an essential hormone for the continuation of pregnancy and is prescribed in $13-40 \%$ of women with threatened miscarriage, according to the literature [8]. Some authors suggested that progesterone reduced the abortion rate in women with threatened miscarriage [7]. Progesterone shows these effects by releasing certain anti-abortive cytokines, modulation of the maternal immune system (immunological tolerance of the fetus), and with relaxation of uterine muscles [9]. Data on the use of progesterone for threatened miscarriage remain conflicting. A more recent systematic review suggested lack of evidence to support routine use of progesterone to prevent miscarriage in early to mid-pregnancy [10].

Recently, Giorlandino et al. [11], speculated that exogenous progesterone use could lead to abnormal blood flow pattern, which in turn may affect fetal development in the first trimester. Also, they concluded that exogenous progesterone increases NT. In our study, we aimed to compare the NT thickness of patients receiving and not receiving progesterone for threatened miscarriage, and evaluate the results of the prenatal screening test.

\section{MATERIAL AND METHODS}

We designed a retrospective study of patients with threatened miscarriage. Local Ethics Committee approved of the study. The archives of our early pregnancy and emergency units were searched to identify patients who met the criterion of vaginal bleeding and were diagnosed with threatened miscarriage in the early first trimester between January 2013 and December 2013. This is a tertiary referral research and education hospital located in the capital city of Turkey, Ankara. Informed consent was not obtained due to retrospective design of the study. The inclusion criteria for the entire cohort were as follows: maternal age of 18-40 years, diagnosis of threatened miscarriage, BMI of $<30 \mathrm{~kg} / \mathrm{m}^{2}$ at diagnosis, non-smoker, no history of co-morbidities or medications other than progesterone, and an ultrasound test performed between $11^{0}$ to $13^{+6}$ and $15^{0}$ to $19^{+6}$ weeks of gestation with antenatal screening test results. We excluded patients with recurrent miscarriage, intrauterine fetal exitus, major fetal malformation, uterine anomaly, multiple pregnancies, and after assisted reproductive techniques. We also excluded patients who had incomplete laboratory data and had no available medical records or ultrasound reports.
Patients were subdivided into the study group (progesterone treated) and controls (non-treated) based on whether they received progesterone treatment. Patients who were treated with oral micronized progesterone (Progestan ${ }^{\circledast}$, Natural Micronized Progesterone, Koçak Farma, Turkey) were identified on the basis of patient files or the official prescription record system. A $300 \mathrm{mg}$ dose of orally administered micronized form per day, a dose regimen recommended by pharmaceutical companies, was considered as standard. All patients from the study group had used progesterone for at least 10 days.

A careful physical and pelvic examination was performed in all patients after the initial evaluation, which included obstetric and general history. It was recorded that all pregnancies continued beyond 20 weeks of gestation. We also confirmed that all NT measurements were performed by certified expert radiologists and/or gynecologists of our institution using a General Electric Logiq A5 (Milwaukee, USA) convex probe $(3.5 \mathrm{~Hz}$ ) or transvaginal probe $(5 \mathrm{~Hz})$. The CRL lengths were kept between 45 to $84 \mathrm{~mm}$ for NT measurements.

The evaluated clinical and laboratory characteristics included: age, BMI, gravidity, parity, number of abortions, smoking status, comorbidities, additional medications, gestational week at diagnosis, progesterone therapy duration, and results of the combined and triple tests, including MoMs of PAPP-A, free $\beta-h C G, A F P, E_{3}$, and hCG.

\section{Statistical analysis}

Statistical analysis was carried out using statistical packages for SPSS 15.0 for Windows (SPSS Inc., Chicago, IL, USA). Mean and standard deviation (SD) were calculated for continuous variables. Chi-square $\left(\mathrm{X}^{2}\right)$ test and Independent Samples t-test evaluated the associations between categorical and continuous variables. Non-normally distributed categorical variables were evaluated by Mann-Whitney $U$ test. Pearson correlation analysis was used to assess correlations between the clinical parameters. ROC curve analysis was used to find the discriminative factors between the groups. Two-sided $\mathrm{P}$ values were considered as statistically significant at $\mathrm{p}<0.05$.

\section{RESULTS}

A total of 192 pregnant women with threatened miscarriage were included in this retrospective comparative study. Ninety-five women treated with progesterone constituted the study group, whereas 97 women who were not treated with progesterone constituted the control group. Table 1 shows the comparison of cases between the two groups with regard to the demographic and clinical characteristics. Statistically significant differences were noted in median gravidity between the groups $(p=0.041)$. Mean gestational age at the 
Table 1. Comparison of cases between two groups according to the demographic and clinical characteristics

\begin{tabular}{|l|c|c|c|}
\hline & Progesterone-treated group (n: 95) & Control group (n: 97) & P value \\
\hline Age (years) & $25.8 \pm 5.5$ & $26.4 \pm 4.6$ & 0.139 \\
\hline Gravidity & $2(1-6)$ & $2(1-4)$ & 0.041 \\
\hline Parity & $1(0-3)$ & $0(0-2)$ & 0.100 \\
\hline Spontaneous abortion & $0(0-3)$ & $0(0-2)$ & 0.238 \\
\hline Elective abortion & $0(0-2)$ & $0(0-1)$ & 0.541 \\
\hline Children alive & $1(0-3)$ & $0(0-2)$ & 0.508 \\
\hline Gestational week & $8.0 \pm 1.7$ & $8.4 \pm 1.8$ & 0.101 \\
\hline Initial week & $8.0 \pm 1.7$ & & \\
\hline Treatment duration (weeks) & $3.9 \pm 1.6$ & & \\
\hline
\end{tabular}

Data are presented as mean \pm SD and median (min-max). $\mathrm{p}<0.05$ is considered statistically significant

Table 2. Distribution of MoM values in two groups

\begin{tabular}{|l|c|c|c|}
\hline & Progesterone-treated group (n: 95) & Control group (n: 97) & P value \\
\hline PAPP-A & $1.04 \pm 0.73$ & $1.13 \pm 0.88$ & 0.388 \\
\hline NT & $1.13 \pm 0.42$ & $0.91 \pm 0.29$ & $<0.001$ \\
\hline Free-hCG & $1.14 \pm 0.66$ & $1.03 \pm 0.76$ & 0.015 \\
\hline E & $0.97 \pm 0.38$ & $0.97 \pm 0.55$ & 0.525 \\
\hline AFP & $1.06 \pm 0.47$ & $0.94 \pm 0.35$ & 0.146 \\
\hline hCG & $1.17 \pm 0.37$ & $1.01 \pm 0.41$ & 0.007 \\
\hline
\end{tabular}

Data are presented as mean $\pm S D$, and values were expressed as multiple of median (MoM). $P<0.05$ is considered statistically significant. PAPP-A - pregnancy associated plasma protein-A, $E_{3}$ - estriol, hCG — human chorionic gonadtropin, AFP — alfa-feto protein, NT — fetal nuchal translucency

Table 3. Comparison of the prenatal assessment of risk of fetal aneuploidy

\begin{tabular}{|c|c|c|c|}
\hline & Progesterone-treated group ( $\mathrm{n:} 95$ ) & Control group (n: 97) & P value \\
\hline $\begin{array}{l}\text { Biochemical risk } \\
\quad<1 / 50 \\
1 / 50-1 / 100 \\
1 / 100-1 / 250 \\
>1 / 250\end{array}$ & $\begin{array}{c}0 \\
1 \\
2 \\
91\end{array}$ & $\begin{array}{c}0 \\
2 \\
1 \\
93\end{array}$ & 0.615 \\
\hline $\begin{array}{l}\text { Double risk } \\
\quad<1 / 50 \\
1 / 50-1 / 100 \\
1 / 100-1 / 250 \\
>1 / 250\end{array}$ & $\begin{array}{c}0 \\
3 \\
5 \\
87\end{array}$ & $\begin{array}{c}0 \\
2 \\
5 \\
90\end{array}$ & 0.891 \\
\hline $\begin{array}{l}\text { Combined risk } \\
<1 / 50 \\
1 / 50-1 / 100 \\
1 / 100-1 / 250 \\
>1 / 250\end{array}$ & $\begin{array}{c}0 \\
0 \\
0 \\
95\end{array}$ & $\begin{array}{c}0 \\
0 \\
2 \\
95\end{array}$ & 0.497 \\
\hline
\end{tabular}

Data are presented as number

initial diagnosis was $8.0 \pm 1.7$ and $8.4 \pm 1.8$ weeks in the study and control groups, respectively $(p=0.101)$. Mean duration of treatment was $3.9 \pm 1.6$ weeks in the progesterone-treated group. Mean MoM value of the NT thickness was statistically significantly higher in the study group, and mean serum levels of free $\beta$-hCG and hCG were also higher in this group $(p<0.05)$ (Table 2). The results of the risk assessment in both, combined and triple test, revealed no statistically significant differences between the two groups (Table 3). When all variables were included in the ROC curve analysis, it was demonstrated that only NT, hCG, and free $\beta$-hCG (AUC: 0.666, p < 0.001, 95\% Cl: 0.587-0.742; AUC: 0.636, $p=0.001,95 \%$ Cl: 0.557-0.716, AUC: $0.600, p=0.015,95 \% \mathrm{Cl}: 0.519-0.681$, respectively) were discriminative factors for women receiving progesterone for 


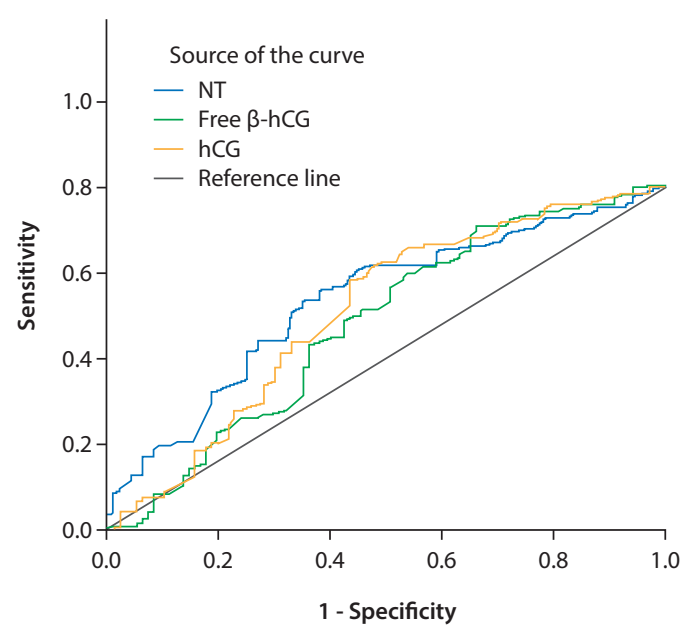

Figure 1. ROC curve analysis of significant discriminants for the progesterone treated group

threatened miscarriage (Figure 1). Also, there was a positive correlation between NT and treatment duration $(r=0.269$; $\mathrm{p}<0.001)$.

\section{DISCUSSION}

In our study, we intended to compare NT thickness of patients receiving and not receiving progesterone for threatened miscarriage, and to evaluate whether oral progesterone therapy increases NT thickness and causes abnormal antenatal screening test results. Our results revealed NT thickness to be higher in patients who were treated with oral progesterone. Also, this treatment does not cause abnormal antenatal screening test results.

Progesterone is a steroid hormone which plays a crucial role at each stage of pregnancy, and modulates maternal immune response to prevent rejection of the embryo. Progesterone regulates the peri-implantation period and immune responses during pregnancy, but also suppresses uterine contractions during pregnancy, and initiates labor and cervical ripening at the end of pregnancy. In the human reproductive system, progesterone exerts its effects via genomic and non-genomic actions which converge to produce tissue- and cell-specific responses [12].

Trophoblastic invasion is strictly controlled by proteolytic enzymes such as matrix metalloproteinase (MMP) which digest extracellular matrix components, e.g. collagen. Progesterone acts as a regulator of trophoblast invasion by controlling MMP activity directly or indirectly via local mediators, e.g. cytokines [13].

Several studies have suggested that progesterone decreases spontaneous miscarriages and increases implantation rates. Although there is no evidence to support the routine use of progestagens for the treatment of threatened miscarriage, progestagens are often used in routine practice due to patient expectations to receive prescriptions. Also, the optimal route of administration and the dose regimen remain unclear. According to a limited number of meta-analyses, it was suggested that progesterone, both in prevention or treatment of miscarriages, does not change the outcome of pregnancy. Data on the potential harms to the mother and/or the child connected with the use of progestagens are scant [14]. Therefore, the use of progesterone during the first trimester of pregnancy should be reserved to cases of assisted reproductive technology pregnancies, where the luteal phase is insufficient, and in cases of women at risk for preterm birth $[15,16]$.

It is universally known that hCG belongs to the family of glycoprotein hormones characterized by a heterodimeric structure, with the $a$ and $\beta$-subunits. The function of hCG is still defined as 'progesterone promotion' in most medical textbooks, but we also know that hCG has numerous other important placental, uterine, and fetal functions in pregnancy. After embryo implantation, hCG starts to be produced by trophoblast and interacts with the LH (luteinizing hormone)/hCG receptor of the ovary and promotes the maintenance of the corpus luteal progesterone production. This continues for approximately 4 weeks. After that time, there are sufficient syncytiotrophoblast cells in the placenta to take over progesterone production from the corpus luteum [17]. In a previous study performed with cultured human cells, it was shown that progesterone suppressed hCG secretion, and suppression of hCG by progesterone occurred in a dose-response manner [18]. Interestingly, we found higher serum hCG levels in women treated with progesterone in both, combined and triple test results. This situation may be due to differences in the risk of miscarriage or amount of bleeding.

Increased NT thickness may not always be associated with fetal chromosomal abnormalities. Although different proposals exist in the literature (e.g. cardiac dysfunction, venous congestion, and delayed or abnormal lymphatic drainage), the real pathophysiological mechanism remains to be fully elucidated [19]. On the other hand, the effect of progesterone has been attempted to be explained by hemodynamic disturbance that leads to fluid accumulation [11]. Regardless, we are of the opinion that progesterone may also increase the NT by changing the ultra-structure of the extracellular matrix.

To the best of our knowledge, this study has been one of the first to investigate the relation between progesterone therapy and NT thickness. A recent study by Giorlandino et al. [11], showed that exogenous progesterone therapy affects fetal NT thickness regardless of progesterone content, dosage, and route of administration. These authors had important confounders, including gestational age, maternal age, BMI, smoking status in their large numbered prospec- 
tive study but they found a strong association between the use of progesterone and NT thickness in all the gestational ages considered, with a higher degree of significance at $11^{0}$ to $11^{+6}$ weeks of gestation after controlling these confounders. In our study, mean MoM of NT thickness was significantly higher in the progesterone-treated group, what was consistent with their report. Our study had several limitations, including its retrospective nature. Also, the patients were not divided into different categories according to their gestational weeks. Regardless, the groups were homogenously distributed, and we only included women who had threatened miscarriage and were treated with oral natural progesterone.

\section{CONCLUSIONS}

Our study confirms that oral progesterone therapy for threatened miscarriage in the first trimester may increase fetal NT thickness and may alter maternal serum HGC levels. However, it seems that it was not associated with abnormal prenatal screening test results. There might be a need for readjusting the risk in patients who received progesterone treatment in the early pregnancy. Large sample sizes, double-blind and randomized controlled trials are needed to detect the exact effects of progesterone on fetal screening tests.

\section{Conflict of interest}

The authors declare no conflict of interest.

\section{Funding information}

The authors have received no funding for this article.

\section{REFERENCES}

1. Wald NJ. The triple test. Clinical Chemistry. 2014, 60, 269-270.

2. Wald NJ.The detection of neural tube defects by screening maternal blood. In: Boué A (ed.). Prenatal diagnosis. Paris: Colloques de INSERM 1976;61:227-238.

3. Wald NJ, Cuckle HS, Densem JW, [et al.]. Maternal serum screening for Down's syndrome in early pregnancy. BMJ 1988, 297 (6653), 883-887.

4. Haddow J, Palomaki G, Knight G, [et al.]. Screening of maternal serum for fetal Down's syndrome in the first trimester. NEnglJMed. 1998, 14, 995-1061.

5. Nicolaides $\mathrm{KH}, \mathrm{Azar} \mathrm{G}$, Byrne D, [et al.]. Fetal nuchal translucency: ultrasound screening for chromosomal defects in first trimester of pregnancy. BMJ. 1992, 304 (6831), 867-869.

6. Bianchi DW, Parker RL, Wentworth J, [et al.]; CARE Study Group. DNA sequencing versus standard prenatal aneuploidy screening. $N$ Engl J Med. 2014, 370 (9), 799-808.

7. Yassaee F, Shekarriz-Foumani R, Afsari S, [et al.]. The effect of progesterone suppositories on threatened abortion: a randomized clinical trial. J Reprod Infertil. 2014, 15 (3), 147-151.

8. Sotiriadis A, Papatheodorou S, Makrydimas G. Threatened miscarriage: evaluation and management. BMJ. 2004, 329 (7458), 152-155.

9. Halasz M, Szekeres-Bartho J. The role of progesterone in implantation and trophoblast invasion. J Reprod Immunol. 2013, 97 (1), 43-50.

10. Haas DM, Ramsey PS. Progestogen for preventing miscarriage. Cochrane Database Syst Rev. 2013, 10, CD003511.

11. Giorlandino $C$, Cignini P, Padula F et al. Effects of exogenous progesterone on fetal nuchal translucency: an observational prospective study. Am J Obstet Gynecol. 2015, 212 (3), 335.e1-7.

12. Gellersen B, Fernandes MS, Brosens JJ. Non-genomic progesterone actions in female reproduction. Hum Reprod Update. 2009, 15, 119-138.

13. Staun-Ram E, Shalev E. Human trophoblast function during the implantation process. Reprod Biol Endocrinol. 2005, 3, 56.

14. Dante G, Vaccaro V, Facchinetti F. Use of progestagens during early pregnancy. Facts Views Vis Obgyn. 2013, 5 (1), 66-71.

15. van der Linden $M$, Buckingham $K$, Farquhar $C$, [et al.]. Luteal phase support for assisted reproduction cycles. Cochrane Database Syst Rev. 2011, 10, CD009154.

16. Dodd JM, Jones L, Flenady V, [et al.]. Prenatal administration of progesterone for preventing preterm birth in women considered to be at risk of preterm birth. Cochrane Database Syst Rev. 2013, 7, CD004947.

17. Cole LA. Biological functions of hCG and hCG-related molecules. Reprod Biol Endocrinol. 2010, 8, 102.

18. Wilson EA, Jawad MJ, Powell DE. Effect of estradiol and progesterone on human chorionic gonadotropin secretion in vitro. Am J Obstet Gynecol. 1984,149 (2), 143-148.

19. Nicolaides $\mathrm{KH}$, Heath $\mathrm{V}$, Cicero $\mathrm{S}$. Increased fetal nuchal translucency at 11-14 weeks. Prenat Diagn. 2002, 22, 308-315. 